\title{
An Improved Model for Antennas in Reverberation Chambers
}

\author{
John M. Ladbury, David A. Hill \\ National Institute of Standards and Technology \\ 325 Broadway MS 818.02, Boulder, CO 80305 USA \\ john.ladbury@nist.gov
}

\begin{abstract}
We present an improved model for antennas in a reverberation chamber. The derivation of this model is more rigorous than previous derivations and models, but is still, admittedly, overly simplistic. However, this model should be applicable for a special class of antennas (those that can be modeled as an unknown 2-port network between the test port and an ideal antenna). For more realistic antennas, these models should still provide valuable insight into the possible behavior of any antenna. Using these models, we show that some previous assumptions regarding receiving characteristics (mismatch and efficiency) of an antenna are invalid: most significantly we show that there can be differences between the transmitting and receiving efficiency of an antenna, and also between the transmitting and receiving mismatch factors. However, we show that the errors are unimportant for most measurements. We also give justification for the assumption that the average reflection coefficient of an antenna in a reverberation chamber is simply the free-space reflection coefficient of the antenna.
\end{abstract}

\section{INTRODUCTION}

We present improved models for antennas in a reverberation chamber. When we first encountered measurement issues related to antennas in a reverberation chamber (efficiency, transmission impedance mismatch, reception impedance mismatch) [1], we turned to standard definitions [2]. We found a flow chart defining parts of a transmitting antenna, but for transmitting antennas we could only find the vague statement "A similar flow chart can be constructed for a receiving antenna." From this, we developed some very simplistic approximations and models. We assumed that the reception impedance mismatch factor $m_{R}$ was identical to that of the transmission impedance mismatch factor $m_{T}$, and that the impedance mismatch factors $m_{R}$ and $m_{T}$ for an antenna in a reverberation chamber were essentially the same as impedance mismatch factors $m_{R f s}$ and $m_{T f s}$ for that same antenna in free space. We also assumed that, for a reciprocal antenna, the receiving and transmitting antenna efficiencies are equal, that is $\eta_{R}=\eta_{T}$. These are common assumptions in antenna theory [3]. Although these approaches made measured data "look" better, we had little more than a few simple "hand-waving" arguments and a few measurements to support them. Such an approach is intuitively satisfying, but makes estimation of uncertainties complicated.

Ideally, a complete model for an antenna in a reverberation chamber could be constructed by modeling the antenna as a multiport network, with one port being the connector on the antenna, and each of the remaining ports assigned to a possible mode or ray path inside the chamber. This would obviously be a complicated and cumbersome approach. Some simplification may be possible that would take into account the statistical nature of a reverberation chamber, similar to what is done for ideal antennas in an ideal chamber [4], but the required method is not obvious. For this report, we will use a less sophisticated but still powerful approach. We begin with a very simple model of an antenna, discuss what we expect and know about it when the antenna is placed in free space, and then investigate what happens when we use that model in an ideal reverberant environment.

\section{BASIC MODEL AND NOTATION}

Our basic model is shown in Figure 1. Starting with any simple antenna with a single port, Port 1, we model this antenna as a perfect antenna (lossless and perfectly matched) connected to an unknown 2-port network. We can assume that the pattern and scattering characteristics of our ideal antenna are the same as those of our real antenna. The 2-port network will model the imperfections (mismatch and loss) of the real antenna. The network is attached to the ideal antenna via a virtual port, Port 2 . For simplicity, we will assume that the intrinsic impedance $Z_{0}$ of Port 2 is the same as for Port 1 , or $Z_{01}=Z_{02}$. For most applications this will be $50 \mathrm{ohms}$. If we then separate the 2-port from the ideal antenna, we can refer to the output port of the 2-port as Port 2, and the input of the ideal antenna as Port $2 \beta$. We will assume that the 2-port is reciprocal, that is, $S_{12}=S_{21}$.

Ideally, we would simply throw away the 2-port and attach directly to Port $2 \beta$ of the perfect antenna. Alas, this is not allowed. So instead, we examine the characteristics of the 2port that will give us a model that most closely resembles the real antenna. To do this, we first examine how such an antenna, with an arbitrary network, would behave in free space.

If we transmit with our antenna then there will be no reflections from the ideal antenna in free space, so our 2-port will appear to be terminated by a non-reflecting load. The reflection coefficient of the antenna will simply be the reflection coefficient $S_{11}$ of the 2-port, and the transmission mismatch factor $m_{T}$ will be $1-\left|S_{11}\right|^{2}$. For an incident power of $P_{\text {inc }}$, the reflected power will be $P_{\text {inc }}\left|S_{11}\right|^{2}$, the net power into the antenna will be $P_{\text {inc }}\left(1-\left|S_{11}\right|^{2}\right)$, and the power into the ideal antenna, which is the transmitted power, will be $P_{\text {inc }}\left|S_{21}\right|^{2}$. 
From this, the transmitting efficiency $\eta_{T}$ of the antenna will simply be the transmitting efficiency of the 2-port terminated in a non-reflecting load, or

$$
\eta_{T}=\frac{\left|S_{21}\right|^{2}}{1-\left|S_{11}\right|^{2}}
$$

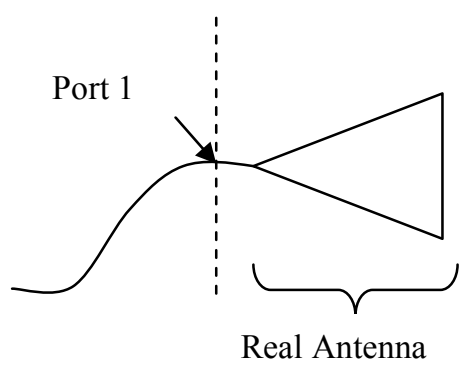

(a)

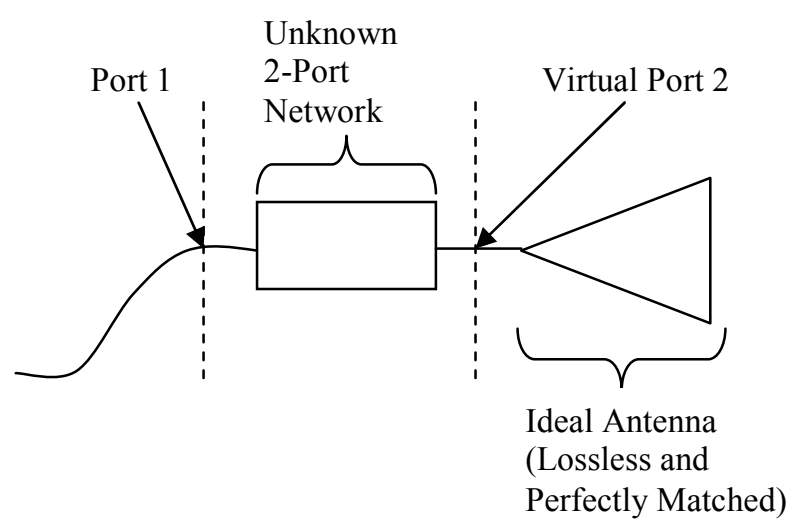

(b)

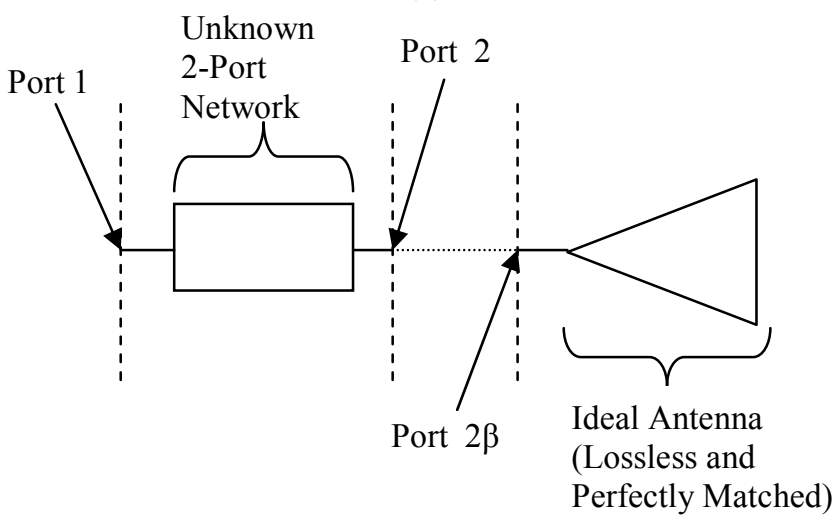

(c)

Figure 1. Antenna, model, and notation. (a) Original antenna, (b) simplified model, (c) the unknown 2-port, and (d) the ideal lossless and perfectly matched antenna.

Equations similar to (1) appear often in network analysis [5], and also in other papers related to reverberation chambers [1].
However, in those papers, Ports 1 and 2 generally refer to the ports of transmitting and receiving antennas, and should not be confused with the meaning in this paper.

When used as a receiving antenna, we will assume that the receiving instrument attached to Port 1 also represents a nonreflecting load. Receiving characteristics of an antenna are a little more complicated than transmitting characteristics. Every incident plane wave will result in scattering in all directions, and a signal that is received by the antenna and reflected by the 2-port will also be scattered (re-transmitted) in all directions. As a result, discussing the receiving reflection coefficient of the antenna is somewhat ambiguous, and phase angles have very little meaning. However, we can say that for a signal received by the antenna with power $P_{\text {rec }}$, the power reflected by the 2-port, and therefore by the antenna is $P_{\text {rec }}\left|S_{22}\right|^{2}$, the reception mismatch factor $m_{R}$ is $1-\left|S_{22}\right|^{2}$, the net power into the antenna will be $P_{\text {rec }}\left(1-\left|S_{22}\right|^{2}\right)$, and the power into the receiving instrument is $P_{\text {meas }}=P_{\text {rec }}\left|S_{12}\right|^{2}$, or, given reciprocity of the 2-port, $P_{\text {meas }}=P_{\text {rec }}\left|S_{21}\right|^{2}$. From this, the receiving efficiency $\eta_{R}$ of the antenna will simply be the receiving efficiency of the 2 -port terminated in a perfect load, or

$$
\eta_{R}=\frac{\left|S_{21}\right|^{2}}{1-\left|S_{22}\right|^{2}} .
$$

From the above analysis, a reasonable first step to model a real antenna is to assume that $S_{11}$ for our 2-port is simply the same as $S_{11 f s}$ for our real antenna when placed in free space.

We can use the efficiency relationship in Eq. (1) or Eq. (2) to determine the magnitudes $\left|S_{12}\right|$ or $\left|S_{21}\right|$, but efficiency is not generally known or provided by manufacturers, and can be difficult to measure. One possibility is to estimate the efficiency using a reverberation chamber [1][4], but this application is not yet well developed. The phase angle $\varphi_{21}$ is, in general, unknown and will have little impact on the analysis that follows.

The remaining parameter $S_{22}$ is significantly more complicated. In the past [1] we have assumed that $\left|S_{22}\right|=\left|S_{11}\right|$ because it was simple and gave results that were intuitively satisfying. However, if we assume that the unknown 2-port can be any reciprocal and realizable network (and there is no reason to assume otherwise), then we encounter a substantial problem. The magnitudes of the reflection coefficients $\left|S_{11}\right|$ and $\left|S_{22}\right|$ of a general 2-port network do not need to be identical, and neither do the efficiencies $\eta_{1}$ and $\eta_{2}$ [5]. As a result, a general antenna can have significantly different transmission and reception properties, and we cannot simply refer to the efficiency of an antenna, and instead must refer specifically to the transmitting or receiving efficiency. This would seem to imply that general antennas are not necessarily reciprocal. So, if our assumptions regarding the transmission and reception characteristics of an antenna were not valid, how far off can they be, and how does this affect our previous research and results? Fortunately, although the differences can be large, the impact is negligible, as we will show below. 


\section{REVISITING THE ASSUMPTIONS}

We will use a few examples to illustrate some possible differences between transmission and reception characteristics. Starting with one of the simplest cases of a lossless 2-port (perfectly efficient but possibly mismatched antenna), $\left|S_{11}\right|$ and $\left|S_{22}\right|$ will be identical [5], which implies $\eta_{R}=\eta_{T}$. So far, so good. However, if we allow both mismatch and loss, the results are different. One possible way to construct such a network with a specific reflection coefficient and efficiency is to start with a mismatched lossless 2-port with reflection coefficient $S_{11}$ (which gives $\left|S_{21}\right|^{2}=1-\left|S_{11}\right|^{2}$ ), and then attach a perfectly matched attenuator with a power attenuation factor of $\eta_{T}$. This will give the desired transmission characteristics. Looking from the other direction, the magnitude of the reflection coefficient will be $\eta_{T}\left|S_{11}\right|$, and the reception efficiency will be $\left|S_{21}\right|^{2} /\left[1-\left(\eta_{T}\left|S_{11}\right|\right)^{2}\right]$. Obviously, if $\eta_{T}$ is small (much less than unity) and $\left|S_{11}\right|$ is large (very near unity), the differences can be dramatic. And this is just one possible solution, not necessarily the worst possible solution (the extreme cases will be addressed below). But how do these potential differences affect measurements and chamber evaluations?

Hill [4] shows that, for an incident power $P_{\text {inc }}$, the transmitted power is given by

$$
P_{\text {trans }}=P_{\text {inc }} m_{T} \eta_{T}
$$

and for our model,

$$
m_{T} \eta_{T}=\left(1-\left|S_{11}\right|^{2}\right) \frac{\left|S_{21}\right|^{2}}{1-\left|S_{11}\right|^{2}}=\left|S_{21}\right|^{2} .
$$

Similarly, if $P_{\text {rec }}$ is the power that would be received by an ideal antenna, the power $P_{\text {meas }}$ measured by an impedance matched instrument at the antenna terminal is

$$
P_{\text {meas }}=P_{\text {rec }} m_{R} \eta_{R}
$$

and again for our model

$$
m_{R} \eta_{R}=\left(1-\left|S_{22}\right|^{2}\right) \frac{\left|S_{12}\right|^{2}}{1-\left|S_{22}\right|^{2}}=\left|S_{12}\right|^{2}=\left|S_{21}\right|^{2} .
$$

So, even though $m_{R}$ can be significantly different from $m_{T}$ and $\eta_{R}$ can be significantly different from $\eta_{T}$, assuming they are equal introduces no error as long as we are always dealing with the product of the impedance mismatch factor and the efficiency.

\section{ANTENNA MODELS IN A REVERBERATION CHAMBER}

Thus far we have evaluated the behavior of our antenna model in free space. We now examine what happens once such a model is placed in an ideal reverberation chamber. To do this we begin with the statistical behavior of an ideal antenna in an ideal chamber. For this situation, the received signal is assumed to have real and imaginary components that are independent and normally distributed with mean 0 and identical variances $\sigma^{2}$ [1]. This statistical model has at least one obvious flaw: a normal distribution has an infinite range, but received power is constrained to be less than or equal to the transmitted power. However, if the losses are sufficiently large such that $\sigma^{2}<<1$, which is roughly equivalent to assuming that the antennas are not the dominant loss mechanism in the chamber, then this model appears to be quite good. The statistical model given above is equivalent to assuming that the phase angle of the received signal is uniformly distributed between 0 and $2 \pi$ (that is, all phase angles are equally likely), the magnitude has a Rayleigh distribution, and the magnitude and phase are independent.

Placing our receiving antenna model inside of a reverberation chamber with the given characteristics is equivalent to connecting port 2 of our 2-port network to a random source having the statistical properties described above: the phase angle is uniformly distributed between 0 and $2 \pi$ and the magnitude has a Rayleigh distribution. The measured signal at Port 1 will have the same statistical properties but will be scaled by $\left|S_{21}\right|$. This is consistent with previous analysis [1][4].

If we transmit with this antenna, the reflection coefficient observed at Port $2 \beta$ will have the same general statistical properties, although the variance of the real and imaginary components will be $2 \sigma^{2}$ [6]. This is equivalent to terminating our 2-port network with a load having a random reflection coefficient $\Gamma_{L} . \Gamma_{L}$ will have the statistical properties described above: the phase angle is uniformly distributed between 0 and $2 \pi$, and the magnitude has a Rayleigh distribution. The reflection coefficient $\Gamma_{1}$ looking into Port 1 of our 2-port network terminated by such a random load will also be random, and we now examine the statistical characteristics of the results.

For a given $\Gamma_{L}$ with magnitude $r$ and phase $\varphi_{L}, \Gamma_{1}$ is given by [5]

$$
\Gamma_{1}=S_{11}+\frac{S_{12} S_{21} \Gamma_{L}}{1-S_{22} \Gamma_{L}}=S_{11}+\frac{S_{12} S_{21}}{\frac{1}{|r|} e^{-j \varphi_{L}}-S_{22}} .
$$

Since the magnitude and phase are assumed to be independent, it is useful to examine the effects of changing the magnitude and phase of $\Gamma_{L}$ separately. If we hold the magnitude $r$ constant and vary the phase, this is equivalent to attaching a sliding termination to our 2-port network, and this problem has been studied in detail [5]. Equation (7) will transform the $\Gamma_{L}$ circle with radius $r$ centered at the origin to a new circle with radius

$$
R_{1}=\frac{\left|S_{12} S_{21} \Gamma_{L}\right|}{1-\left|S_{22} \Gamma_{L}\right|^{2}}
$$

and center located at 


$$
C_{1}=S_{11}+R_{1}\left|S_{22} \Gamma_{L}\right| e^{j\left(\varphi_{12}+\varphi_{21}-\varphi_{22}\right)} .
$$

Figure 2 shows the transformation of several such circles in the $\Gamma_{L}$ plane in Figure 2a, and how they are transformed to the $\Gamma_{1}$ plane in Figure $2 \mathrm{~b}$. For these figures we assumed values for $\left|\Gamma_{L}\right|$ from 0 to 1 with a step of 0.1 , a perfectly efficient antenna $\left(\eta_{T}=1\right)$, and a large but reasonable mismatch with $\left|S_{11}\right|=0.8$ and a phase of $45^{\circ}$.

Once again, in Figure $2 b$, we appear to have a problem... we have previously assumed that the average value $\left\langle\Gamma_{1}\right\rangle$ of the reflection coefficient will be the free space reflection coefficient of the antenna, or $S_{11}$. Unfortunately, the circles in Figure $2 \mathrm{~b}$ are not all centered on $S_{11}$. In fact, for $\left|\Gamma_{L}\right|=1$, the circle is centered on the origin. The only ways for $C_{1}$ to equal $S_{11}$ are if $S_{21}=0$ (no transmission...not very interesting), $\Gamma_{L}=$ 0 (free space, again not very useful in a reverberation chamber), or $S_{22}=0$ (desirable, since this implies a perfectly matched receiving antenna, but this cannot be guaranteed). Also, as $\left|\Gamma_{L}\right|$ increases from 0 to 1 , each subsequent circle drifts farther and farther from $S_{11}$, so again it appears that one of the significant assumptions we have made in that past is incorrect. However, if we take 100 evenly spaced phase angles of $\varphi_{L}$ between 0 and $2 \pi$ for each circle, we see in Figure 2c that the transformed samples of $\Gamma_{1}$ are not uniformly distributed, and perhaps the average is indeed close to $S_{11}$. To check this, we first examine the expected value $\mathbf{E}\left(\Gamma_{1}\right)$ of the reflection coefficient rather than the center of each circular locus. For the following analysis we will again assume that the phase $\varphi_{L}$ is uniformly distributed and independent of the distribution of $\left|\Gamma_{L}\right|$, but will assume that $\left|\Gamma_{L}\right|$ has an arbitrary distribution of $f_{r}(r)$. Since $\varphi_{L}$ and $\left|\Gamma_{L}\right|=r$ are independent, we can write the joint distribution

$$
f_{r, \varphi_{L}}\left(r, \varphi_{L}\right)=f_{r}(r) f_{\varphi_{L}}\left(\varphi_{L}\right)=\frac{1}{2 \pi} f_{r}(r), \quad 0 \leq \varphi_{L}<2 \pi, 0 \leq r \leq 1 .
$$

We can find the expected value as

$$
\mathbf{E}\left(\Gamma_{1}\right)=\int_{0}^{1} \int_{0}^{2 \pi} \Gamma_{1}\left(r, \varphi_{L}\right) f_{r, \varphi_{L}}\left(r, \varphi_{L}\right) r d \varphi_{L} d r .
$$

Simplifying, we get

$$
\begin{aligned}
\mathbf{E}\left(\Gamma_{1}\right) & =\frac{1}{2 \pi} \int_{0}^{1} r f_{r}(r) \int_{0}^{2 \pi} \Gamma_{1}\left(r, \varphi_{L}\right) d \varphi_{L} d r \\
& =S_{11}+\frac{S_{12} S_{21}}{2 \pi} \int_{0}^{1} r f_{r}(r) \int_{0}^{2 \pi} \frac{1}{r^{-1} e^{-j \varphi_{L}}-S_{22}} d \varphi_{L} d r
\end{aligned}
$$

Surprisingly, the rightmost term integrates to 0 for $0 \leq|r| \leq 1$ and $S_{22}<1$, which implies that $\mathbf{E}\left(\Gamma_{1}\right)=S_{11}$.

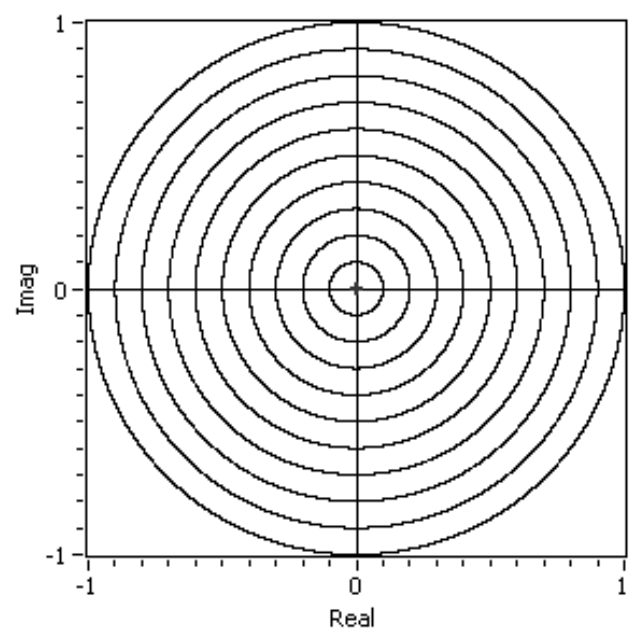

(a)

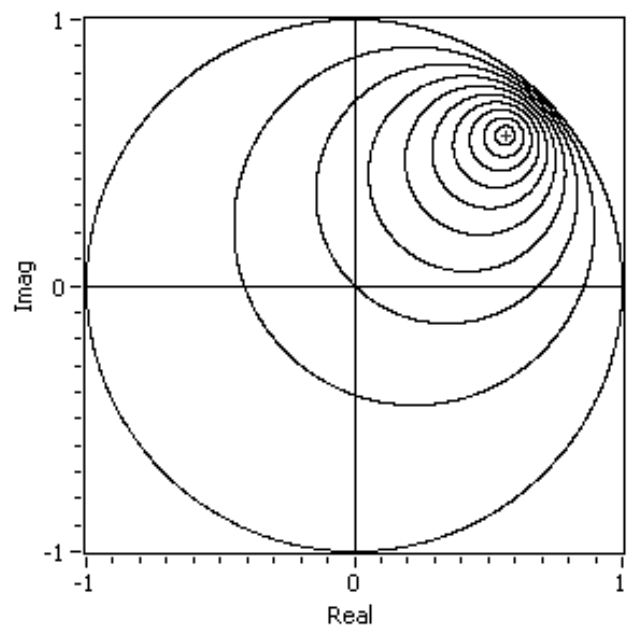

(b)

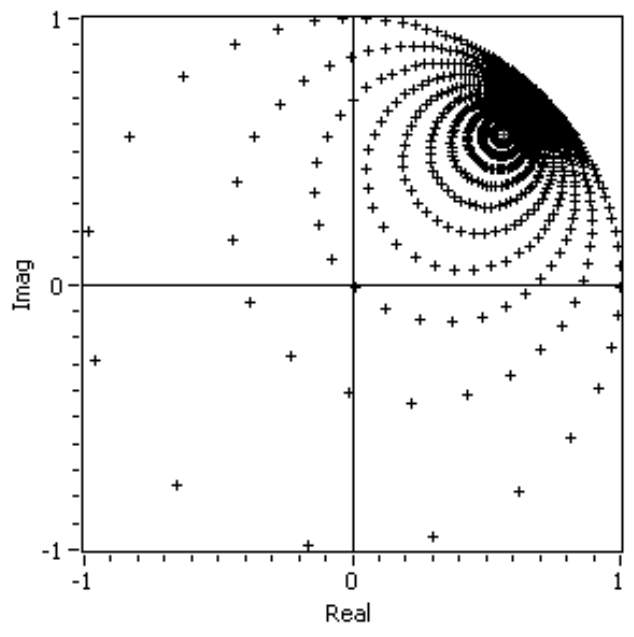

(c)

Figure 2. Transformation of circles from the $\Gamma_{L}$ plane (a) to the $\Gamma_{1}$ plane (b). Uniformly spaced points in the $\Gamma_{L}$ plane are distorted in the $\Gamma_{1}$ plane (c). 


\section{ESTIMATING CHARACTERISTICS OF $S_{22}$}

If the losses in a reverberation chamber are small such that the antennas are the dominant source of loading, errors in estimates of the receiving impedance mismatch factor may cause us to overestimate or underestimate the loading introduced by the antennas. Even though we have shown above that for most situations we can safely assume that $m_{R} \eta_{R}=m_{T} \eta_{T}$, which means we can also assume that $\left|S_{22}\right|=\left|S_{11}\right|$ as long as we also assume that $\eta_{R}=\eta_{T}$, in cases where the losses in the chamber are low, it can still be helpful to determine the possible range for $\left|S_{22}\right|$. To evaluate the possible characteristics of $S_{22}$, we begin with standard realizability constraints [5]. Any passive, linear, time-invariant network must satisfy these constraints. Strict realizability excludes losslessness and we will address the lossless case separately. Assuming both ports of a 2-port network have identical intrinsic impedances, the strict realizability constraints are

$$
\begin{gathered}
0<\frac{\left|S_{21}\right|^{2}}{1-\left|S_{11}\right|^{2}}<1, \\
0<\frac{\left|S_{12}\right|^{2}}{1-\left|S_{22}\right|^{2}}<1, \\
\text { and } \quad\left(1-\left|S_{11}\right|^{2}-\left.\left|S_{21}\right|^{2}|1-| S_{22}\right|^{2}-\left|S_{12}\right|^{2}\right) \\
-\left|\overline{S_{11}} S_{12}+\overline{S_{21}} S_{22}\right|^{2}>0 .
\end{gathered}
$$

If $S_{11}$ and $S_{21}$ are known, and if reciprocity holds, then the values of $S_{22}$ that satisfy the above constraints can be determined through algebraic manipulation. The resulting locus of solutions is contained in a circle inside the $S$ plane with the following constraints:

1. The distance from the center of the $S_{22}$ circle to the origin is $\frac{\left|S_{11}\right|\left|S_{21}\right|^{2}}{1-\left|S_{11}\right|^{2}}=\left|S_{11}\right| \eta_{1}$.

2. The radius of the $S_{22}$ circle is $1-\frac{\left|S_{21}\right|^{2}}{1-\left|S_{11}\right|^{2}}=1-\eta_{1}$.

3. The phase angle $\varphi_{22}$ to the center of the $S_{22}$ circle is $2 \varphi_{21}-\varphi_{11}+\pi$.
For the special case of losslessness, which is equivalent to perfect efficiency or $\eta_{1}=1$, the locus of possible solutions collapses down to a single point: $\left|S_{22}\right|=\left|S_{11}\right|$ as mentioned earlier, and $\varphi_{22}$ is given by constraint 3 above.

For any other efficiency, there is no reason to assume any similarity between $\left|S_{22}\right|$ and $\left|S_{11}\right|$. Possible values for $\left|S_{22}\right|$ are:

$$
\begin{aligned}
0 & \leq\left|S_{22}\right| \leq 1-\eta_{1}\left(1-\left|S_{11}\right|\right), & \eta_{1} \leq 1 /\left(1+\left|S_{11}\right|\right), \\
\eta_{1}\left(1+\left|S_{11}\right|\right)-1 & \leq\left|S_{22}\right| \leq 1-\eta_{1}\left(1-\left|S_{11}\right|\right), & \eta_{1}>1 /\left(1+\left|S_{11}\right|\right) .
\end{aligned}
$$

Actual estimation of $\left|S_{22}\right|$ could be based on measurements of loading introduced by the antennas when placed in a reverberation chamber, but the details will not be given here.

\section{CONCLUSION}

We have presented an improved model for antennas that is particularly well suited for use in reverberation chambers. Use of these models gave several surprising results, including demonstrating that many assumptions we have relied on for previous reverberation chamber analyses were not valid. Fortunately, these same new models showed that the invalid assumptions did not introduce any errors for most typical measurement scenarios. The new models also provided some theoretical basis for the assumption that the average reflection coefficient of an antenna placed in a reverberation chamber is simply the free-space reflection coefficient of that antenna. We have also given equations for determining the possible range for values of the impedance mismatch factor of a receiving antenna in a reverberation chamber.

\section{REFERENCES}

[1] J. Ladbury, G. Koepke, and D. Camell, "Evaluation of the NASA Langley Research Center Mode-Stirred Chamber Facility," U.S. Nat. Inst. Stand. Technol. Tech. Note 1508, 1999.

[2] "IEEE Standard Definitions of Terms for Antennas.," IEEE Std 1451993.

[3] Y.T. Lo and S.W. Lee, Antenna Handbook: Theory, Application, and Design. New York: Van Nostrand Reinhold Company, 1988.

[4] D.A. Hill, Electromagnetic Fields in Cavities: Deterministic and Statistical Theories. New Jersey: Wiley-IEEE Press, 2009.

[5] D.M. Kerns and R.W. Beatty, Basic Theory of Waveguide Junctions and Introductory Microwave Network Analysis. Oxford: Pergamon Press, 1967

[6] J.M. Ladbury and D.A. Hill, "Enhanced Backscatter in a Reverberation Chamber: Inside Every Complex Problem is a Simple Solution Struggling to Get Out," IEEE Int. Symp. Electromagn. Compat., Honolulu, Hawaii, July 2007, pp.1-5. 